\title{
The First Method to Sequence DNA
}

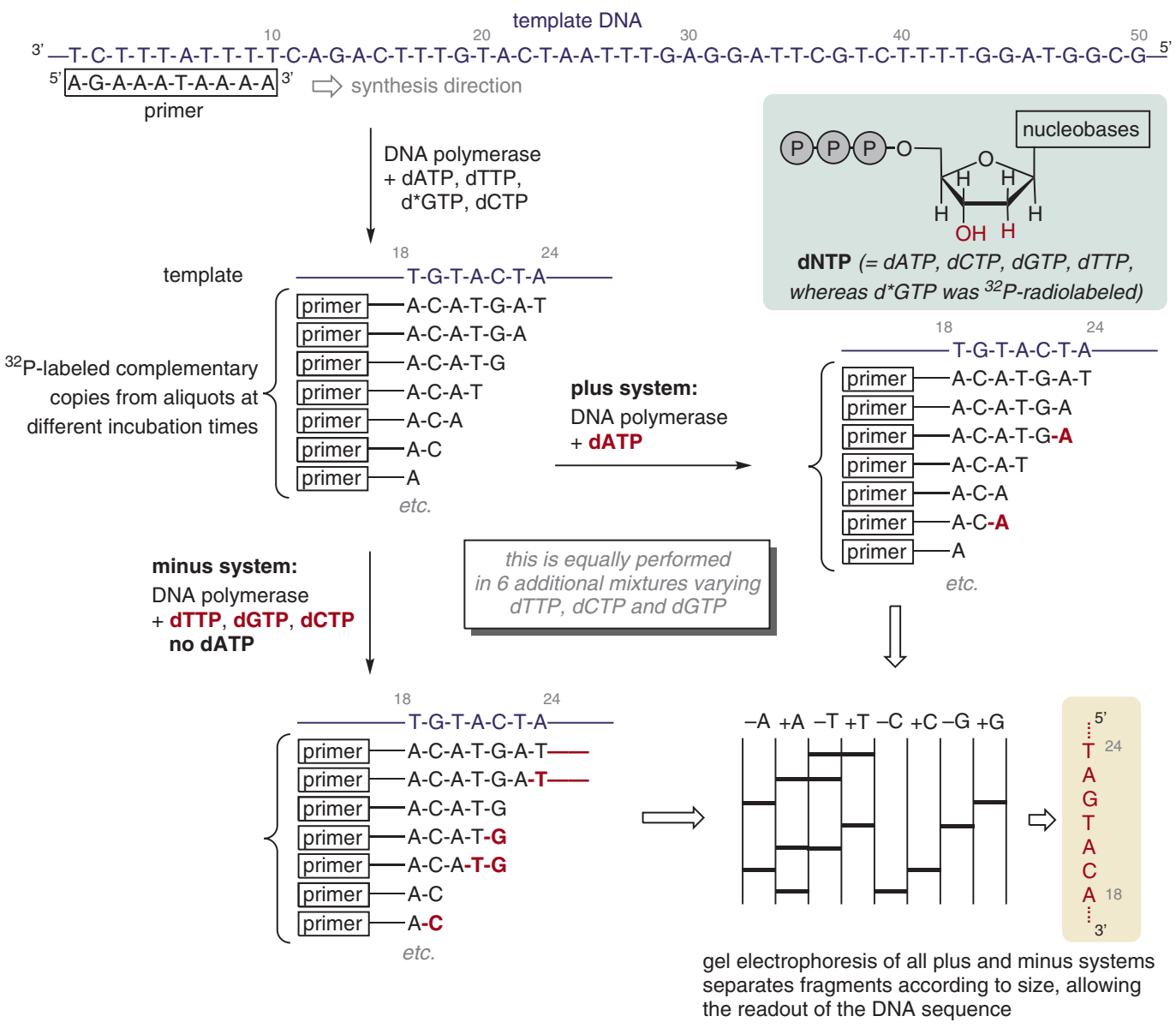

Chemistry in

Medicine and

Biology

Key words

DNA sequencing

DNA polymerase

oligonucleotides

electrophoresis

Syníact

Significance: In 1975, Sanger and Coulson presented the 'plus and minus method' to determine the sequence of a 51 nucleotide, single-stranded DNA. Further development of this idea led to the more commonly known 'chain-terminating method' using dideoxynucleotides (Sanger et al. Proc. Natl. Acad. Sci. U. S. A. 1982, 74, 5463) and automated systems that were vital in the human genome project. Today, the principle of Sanger sequencing remains an important alternative to the next generation methods used to decode DNA sequences.
Comment: A synthetic decanucleotide was used as a primer, enabling DNA polymerase to assemble a complementary strand with free deoxynucleotide triphosphates (dNTPs). Aliquots taken over time were combined and separated from excess dNTPs. Split into a minus and plus system, they were either treated by all but one dNTP or one specific dNTP in the presence of DNA polymerase. This enabled the formation of strands with overlapping nucleotide sequences. Gel electrophoresis separates the complex mixture by size and enables a direct readout of the sequence. 